\title{
Assessment of atrial appendage stasis and thrombosis using MDCT
}

Mecit Kantarci, Recep Sade and Abdurrahim Colak

We read with great interest the Review by Romero et al. (Cardiac imaging for assessment of left atrial appendage stasis and thrombosis. Nat. Rev. Cardiol. 11, 470-480; 2014). ${ }^{1}$ This Review is very useful and draws attention to the important points of imaging stasis and thrombosis in the left atrial appendage. Multidetector computed tomography (MDCT) is widely used for the detection of thrombosis. ${ }^{2}$ Spontaneous echocardiographic contrast (SEC) can mimic thrombosis, but is the result of stasis, which can confuse clinicians. Various

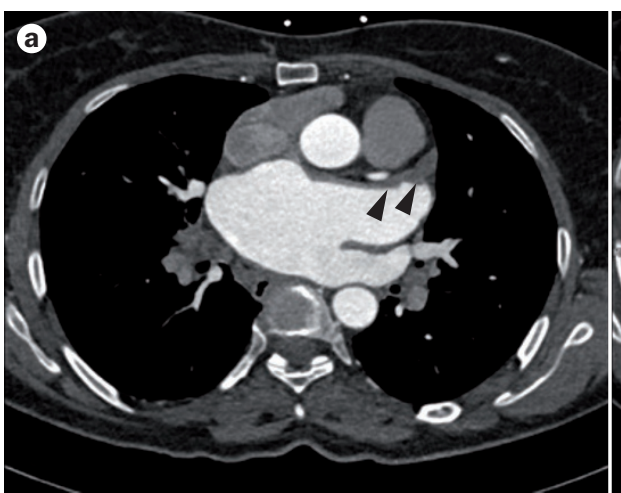

CT techniques can be used to differentiate between stasis and thrombosis, such as biphasic examinations ${ }^{3,4}$ or dual energy applications. ${ }^{5}$ We wish to share our experience of differentiating between stasis and thrombosis using MDCT. In our department, patients who are suspected of having thrombosis or SEC are examined in the prone, rather than supine, position. Using MDCT, SEC can mimic thrombosis when the patient is in the supine position (Figure 1a), but disappears in the prone position (Figure 1b). Diagnosis is highly accurate using this simple manoeuvre.

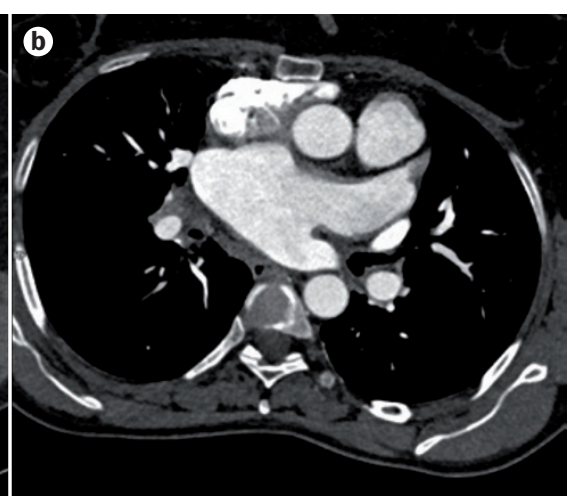

Figure 1 | Differential diagnosis of thrombosis and SEC using multidetector computed tomography. a | When a patient is in the supine position, SEC (arrowheads) can mimic thrombosis. b | However, when the patient is in the prone position, the SEC disappears. Abbreviation: SEC, spontaneous echocardiographic contrast.
Department of Radiology (M.K., R.S.) and Department of Cardiovascular Surgery (A.C.), School of Medicine, Ataturk University, PK 25040, Erzurum, Turkey.

Correspondence to: M.K. akkanrad@hotmail.com

\section{Competing interests}

The authors declare no competing interests.

1. Romero, J., Cao, J. J., Garcia, M. J. \& Taub, C. C. Cardiac imaging for assessment of left atrial appendage stasis and thrombosis. Nat. Rev. Cardiol. 11, 470-480 (2014).

2. Martinez, M. W. et al. Utility of nongated multidetector computed tomography for detection of left atrial thrombus in patients undergoing catheter ablation of atrial fibrillation. JACC Cardiovasc. Imaging 2, 69-76 (2009).

3. Hur, J. et al. Dual-enhanced cardiac CT for detection of left atrial appendage thrombus in patients with stroke: a prospective comparison study with transesophageal echocardiography. Stroke 42, 2471-2477 (2011).

4. Kim, S. C. et al. Differentiation between spontaneous echocardiographic contrast and left atrial appendage thrombus in patients with suspected embolic stroke using two-phase multidetector computed tomography. Am. J. Cardiol. 106, 1174-1181 (2010).

5. Hur, J. et al. Cardioembolic stroke: dual-energy cardiac CT for differentiation of left atrial appendage thrombus and circulatory stasis. Radiology 263, 688-695 (2012). 